Tropical Journal of Pharmaceutical Research September 2021; 20 (9): 1881-1886

ISSN: $1596-5996$ (print); 1596-9827 (electronic)

(C) Pharmacotherapy Group, Faculty of Pharmacy, University of Benin, Benin City, 300001 Nigeria

Original Research Article

http://dx.doi.org/10.4314/tjpr.v20i9.14

\title{
MiR-140-5p inhibits morphine tolerance in rats by targeting TLR4
}

\author{
Guihua Liu, Ying Xiong* \\ Department of Anesthesia, The Second Affiliated Hospital of Dalian Medical University, Dalian City, Liaoning Province 116023, \\ China \\ *For correspondence: Email: xiongying_668@163.com; Tel: +86-0411-84671291-5066
}

\begin{abstract}
Purpose: To determine the influence of miR-140-5p on morphine tolerance in rats. Methods: Sprague-Dawley (SD) rats were randomly divided into morphine tolerance (MT) and saline control (NS) groups, respectively. Rats in MT group were injected with $10 \mu \mathrm{L}(10 \mu \mathrm{g})$ morphine twice daily for seven consecutive days while those in NS group were administered the equivalent volume of normal saline. The maximum effect of morphine (MPE) was computed from tail-flick test results. MiR140-5p mimics and toll-like receptor 4 (TLR4) lentivirus were transfected separately or co-transfected into model rats. MiR-140-5p and TLR4 expression were determined by quantitative real-time polymerase chain reaction (RT-qPCR) or western blotting. Dual-Iuciferase reporter assay was used to verify the target relationship between miR-140-5p and TLR4.

Results: The expression of miR-140-5p was decreased, while the expression of TLR4 increased in morphine-tolerant rats $(p<0.05)$. TLR4 was a target of miR-140-5p. At 24 and $48 \mathrm{~h}$ after injection, MPE clearly increased and TLR4 expression was reduced under miR-140-5p overexpression or TLR4 knockdown $(p<0.05)$. Moreover, there were no significant changes in MPE or levels of TLR4 when miR-140-5p and TLR4 were co-transfected into morphine-tolerant rats.

Conclusion: MiR-140-5p inhibits morphine resistance in rats via targeted regulation of TLR4 expression. These provide a theoretical basis for the clinical management of morphine tolerance.
\end{abstract}

Keywords: MiR-140-5p, Toll-like receptor 4; Morphine tolerance

\begin{abstract}
This is an Open Access article that uses a funding model which does not charge readers or their institutions for access and distributed under the terms of the Creative Commons Attribution License (http://creativecommons.org/licenses/by/4.0) and the Budapest Open Access Initiative (http://www.budapestopenaccessinitiative.org/read), which permit unrestricted use, distribution, and reproduction in any medium, provided the original work is properly credited.
\end{abstract}

Tropical Journal of Pharmaceutical Research is indexed by Science Citation Index (SciSearch), Scopus, International Pharmaceutical Abstract, Chemical Abstracts, Embase, Index Copernicus, EBSCO, African Index Medicus, JournalSeek, Journal Citation Reports/Science Edition, Directory of Open Access Journals (DOAJ), African Journal Online, Bioline International, Open-J-Gate and Pharmacy Abstracts

\section{INTRODUCTION}

Numerous malignant tumors can cause patients unbearable pain when tumors develop to a certain stage. In addition to the pain caused by the tumor itself, the local compression of intravascular nerves, viscera, or bones by the tumor, the elevation of intracranial pressure caused by tumor metastasis to the brain, and the pathological fracture, obstruction, and pressure sores caused by metastasis to bone can result in pain [1]. Morphine is the primary effective analgesic for patients with advanced cancer, but long-term morphine use can lead to drug tolerance. There is increasing evidence that morphine tolerance is a complex process. Currently, studies on the mechanisms of morphine tolerance are not limited to neurons; 
intraperitoneal injection of morphine can lead to significant morphine tolerance, while causing significant hypertrophy of astrocytes in the spinal cord as well as in the hippocampus [2]. Nonetheless, the specific mechanisms of morphine resistance remain to be further investigated [3].

MicroRNAs function by silencing target genes at the transcriptional and post-transcriptional levels [4]. A study reported that microRNAs are also linked to morphine tolerance [5]. Moreover, miR$140-5 p$ is expressed at low levels in a variety of cancer tissues, and exerts a vital influence in cancer invasion and metastasis [6-8]. However, the influence of miR-140-5p in morphine tolerance and its mechanism have not been reported and are uncertain.

Toll-like receptor 4 (TLR4) is a transmembrane signaling receptor that promotes the synthesis and release of cytokines, mediates systemic immune pathological damage, and promotes resistance to viral infections [9]. TLR4 has recently been identified as a drug target for pain treatment [9]. Li et al. pointed out that morphine promotes the development of morphine tolerance through activation of TLR4 [10]. In addition, other studies have shown that the TLR4 gene is specifically controlled by miR-140-5p [11, 12]. In any case, the regulatory roles of miR-140-5p and TLR4 in the development of morphine tolerance remain to be explored further. Therefore, the present study focused on the level of miR-140-5p expression in morphine tolerance, and preliminarily investigated the effect of miR-140$5 p$-targeted regulation of TLR4 on the development of morphine tolerance.

\section{METHODS}

\section{Animal grouping and treatments}

Male Sprague-Dawley (SD) rats $(n=120)$ were provided by the Department of Laboratory Animal Science of Fudan University (Shanghai, China), and weighed 250 - $300 \mathrm{~g}$. Rats with no signs of infection or movement disorders were selected, and randomly divided into the morphine tolerance (MT) group and saline control (NS) group. In the MT group, $10 \mu \mathrm{L}(10 \mu \mathrm{g})$ morphine (Shenyang First Pharmaceutical Co. Ltd, Shenyang, China) was injected into rats twice daily for seven consecutive days, using the modified Yaksh's intra-sheath cannula method to establish morphine tolerance models [13]. In the NS group, $10 \mu \mathrm{L}$ normal saline was injected into rats at the same time on seven consecutive days, using the same method as the MT group. The remaining 100 SD rats were also injected with $10 \mu \mathrm{L}$ morphine to establish morphine tolerance models for subsequent experiments. Animal experiments followed the Guide for the Care and Use of Laboratory Animals [14] and were approved by the Ethics Committee of the Second Affiliated Hospital of Dalian Medical University (approval no. AEE19089).

\section{Behavioral assessment}

Tails of the rats were placed on the thermal radiometer, the radiation temperature was $54{ }^{\circ} \mathrm{C}$, and the cut-off time was $35 \mathrm{~s}$. After placing for 5 min, the rat flicked the tail when it felt pain, and the machine automatically recorded the flicking time. The tail-flick time before injection was taken as the base time, and the tail-flick time after injection was taken as the effect time. The maximum effect of morphine (M) was computed as in Eq 1.

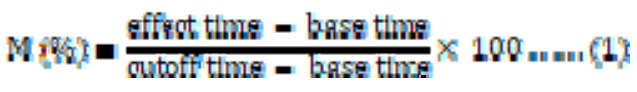

\section{Quantitative real-time polymerase chain reaction (qRT-PCR)}

The intumescentia lumbalis was taken from anesthetized rats to extract the total RNA using TRIzol solution (Sangon Biotech, Shanghai, China). The TransScript All-in-One First-Strand cDNA Synthesis SuperMix for qPCR kit (TransGen Biotech, Beijing, China) was used to detect mRNA expression. The reaction system for qRT-PCR referred to the kit instructions. The primers are listed in Table 1.

Table 1: Primers used for qRT-PCR

\begin{tabular}{lll}
\hline Primer & & Sequence (5' to 3') \\
\hline miR-140-5p & Forward & CTGTGTCCTGCCAGTGGTTTT \\
\multirow{2}{*}{ R6 } & Reverse & CGGTATCCTGTCCGTGGTTCT \\
& Forward & CGCTTACAGCAGACATAC \\
TLR4 & Reverse & TTCACGAATTTGCGTGTCAT \\
& Forward & GGTCAGACGGTGATAGCGAG \\
GAPDH & Reverse & GGATTTCACACCTCCACGCA \\
& Forward & TCGGAGTCAACGGATTTGGT \\
& Reverse & TTCCCGTTCTCAGCCTTGAC \\
\hline
\end{tabular}




\section{Transfection}

After the rats were anesthetized with $350 \mathrm{mg} / \mathrm{kg}$ chloral hydrate, the L5-6 spinous gap of the rat was used as the puncture point, and a vertical distance of $3 \mathrm{~cm}$ between the highest point on the back of rat and the puncture point was maintained for intrathecal catheterization $[15,16]$. Then, the rats were randomly divided into miR$140-5 p$ mimics group, mimics NC group, TLR4 group, miR-140-5p mimics + TLR4 group and empty vector group ( $n=20$ each group). Rats in the miR-140-5p mimics group or mimics NC group were intrathecally injected with $30 \mu \mathrm{L}$ synthetic miR-140-5p mimics or NC mimics. Rats in the TLR4 group or empty vector group were intrathecally injected with $100 \mu \mathrm{L}$ TLR4 lentivirus or empty vector lentivirus, synthesized by Genepharma Co., Ltd. Rats in the miR-140-5p mimics + TLR4 group were intrathecally injected with $30 \mu \mathrm{L}$ miR-140-5p mimics and $100 \mu \mathrm{L}$ TLR4 lentivirus at the same time. For morphine excitation experiments, ten rats in each group were injected with $10 \mu \mathrm{L}(5 \mu \mathrm{g})$ morphine intrathecally before, or 24 or $48 \mathrm{~h}$ after, and then $\%$ MPE was calculated for each group.

\section{Western blotting}

Ten rats from each group were anesthetized before or 24 or $48 \mathrm{~h}$ after injection, and the tissues of intumescentia lumbalis were taken. The tissues were lysed in RIPA lysis buffer (Sangon Biotech) and placed on ice for a while. The protein content of the supernatant was determined using the BCA Protein Assay Kit (Sangon Biotech). Then $35 \mu \mathrm{g}$ total protein was electrophoresed by SDS-PAGE and transferred to PVDF membrane. The membrane with protein was blocked with $2 \%$ bovine serum albumin (BSA) solution (Sangon Biotech), and incubated with TLR4 antibody (1 mg/mL, 1:1500, ab22048, Abcam, Cambridge, USA) overnight at $4{ }^{\circ} \mathrm{C}$. After incubation, the membrane was treated with goat anti-mouse IgG secondary antibody (1 $\mathrm{mg} / \mathrm{ml}, 1: 2000$, ab150120, Abcam) for $1 \mathrm{~h}$. Then, the membrane was developed by adding ECL solution and analyzed using the gel imaging analysis system. The GAPDH antibody was used as a reference.

\section{Dual-luciferase reporter assay}

Cell transfection was performed according to the instructions for Lipofectamine 2000 reagent (Thermo Fisher Scientific). TLR4-wild type (WT) or TLR4-mutant (MUT) plus miR-140-5p mimics were co-transfected into HEK293 cells. The relative luciferase activity was calculated according to the Dual-Luciferase $\AA$ Reporter Assay System protocol (Promega, USA).

\section{Statistical analysis}

All experiments were repeated three times. The data were analyzed using SPSS 22.0 software (SPSS Inc., USA). Measurement data were tested for normal distribution using Shapiro-Wilks (S-W). The comparison of mean differences between multiple groups was processed using analysis of variance (ANOVA), and the correlation between the two groups was analyzed by Spearman's correlation analysis.

\section{RESULTS}

\section{Establishing the morphine tolerance rat models}

The \%MPE of rats in the MT group was clearly increased after morphine injection on days 1, 3, and 5 compared to the NS group ( $p<0.05$, Figure 1). The \%MPE in the MT group gradually weakened from the 1 st to the 7 th days $(p<0.05)$, and on the 7th day, the rats in the MT group showed little difference in \%MPE compared with the NS group. Hence, the above results indicated that the morphine tolerance model was successfully established in rats.

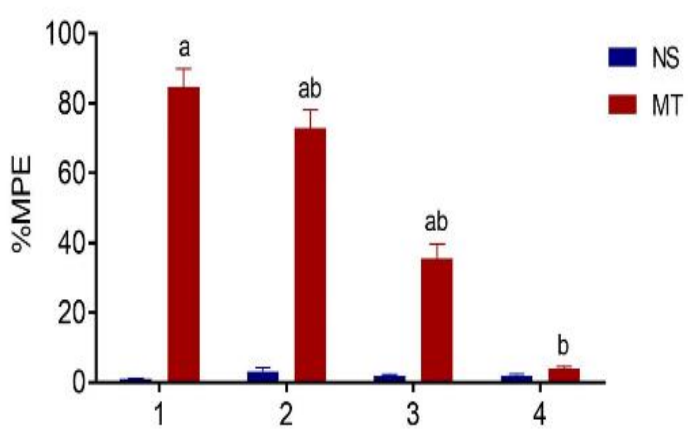

Figure 1: Morphine tolerance of the rats. ${ }^{a} P<0.05$ for MT group compared with NS group; ${ }^{b} p<0.05$ for days 3,5 and 7 compared with day 1

\section{Expression of miR-140-5p and TLR4 in spinal cord of model rats}

RT-qPCR results in Figure 2 show that levels of miR-140-5p in the MT group were decreased, while the expression of TLR4 was significantly higher, than in the NS group. Moreover, the expression level of miR-140-5p was highly negatively correlated with TLR4 $(r=-0.762, p<$ 0.05). 

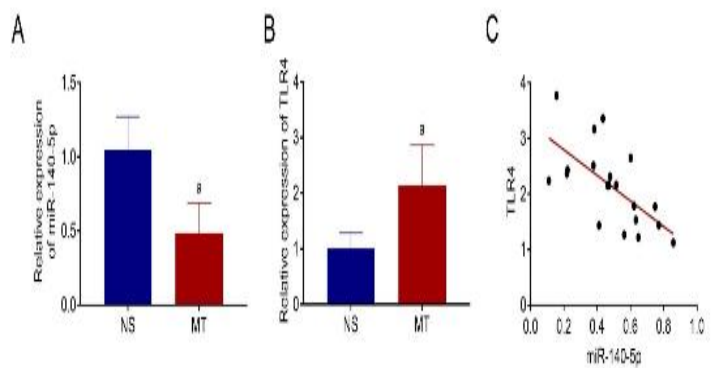

Figure 2: Expression of miR-140-5p and TLR4 in spinal cord of model rats. ${ }^{a} P<0.05$

\section{Effect of miR-140-5p mimics or TLR4 on morphine tolerance in rats}

As shown in Figure 3, there was no significant difference in \%MPE between the groups before injection, but MPE did change significantly 24 and $48 \mathrm{~h}$ after morphine injection in the miR-140$5 p$ mimics group, in comparison to the mimics NC group $(p<0.05)$. In contrast, MPE was significantly reduced by TLR4 overexpression ( $p$ $<0.05)$. However, there was no significant change in MPE of rats in the miR-140-5p mimics + TLR4 group.

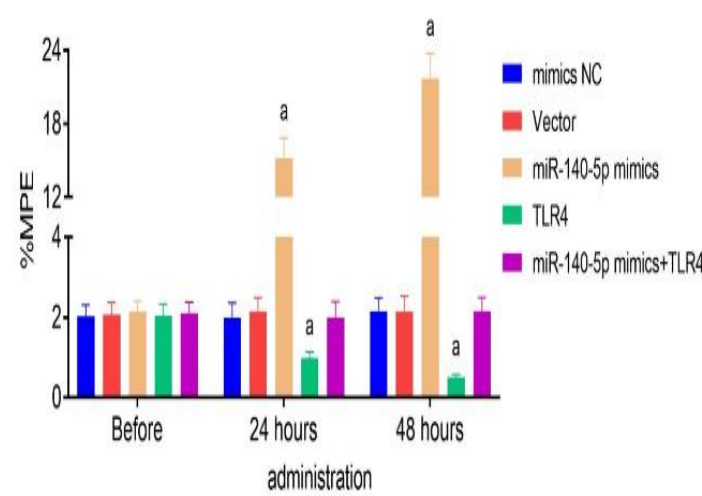

Figure 3: Effects of miR-140-5p mimics or TLR4 on morphine tolerance in rats. ${ }^{a} P<0.05$

\section{Effect of miR-140-5p mimics or TLR4 on TLR4 expression}

Western blotting results in Figure 4 showed that there were no differences in TLR4 expression among the groups before injection. TLR4 expression level was reduced in the miR-140-5poverexpressing group and was increased in the TLR4 group when compared with the empty vector group $(p<0.05)$. However, there was no significant change in TLR4 expression in the miR-140-5p mimics + TLR4 group. In summary, the injection of miR-140-5p mimics in morphinetolerant rats could target the expression of TLR4.

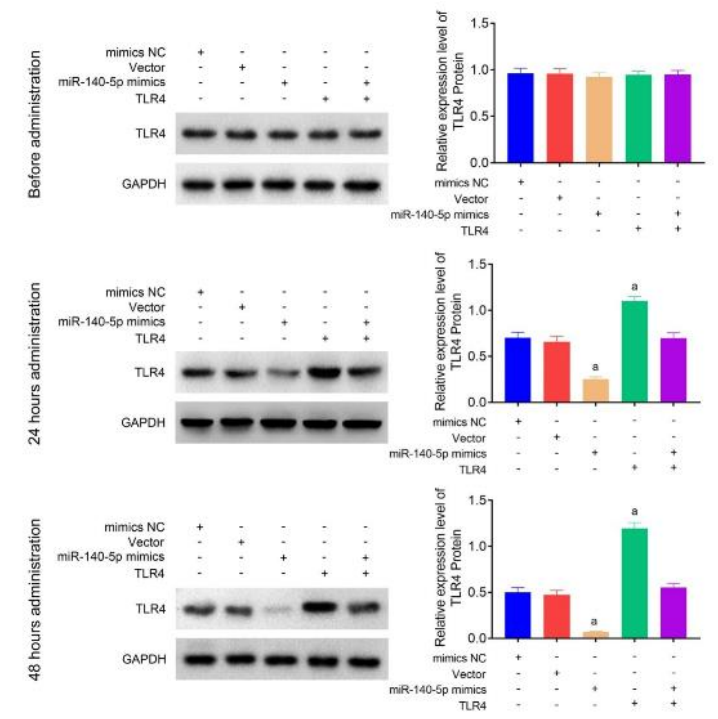

Figure 4: Effect of miR-140-5p mimics or TLR4 on TLR4 expression. MiR-140-5p mimics, NC mimics, TLR4 lentivirus, or empty lentiviral vector were injected into model rats. The TLR4 expression was measured by western blotting before injection, and at 24 and 48 $\mathrm{h}$ after morphine injection

\section{TLR4 was a target of miR-140-5p}

A binding site for miR-140-5p in TLR4 was predicted on the Targetscan website (www.targetscan.org), as shown in Figure 5 A. Dual-luciferase reporter assay results in Figure 5 B show that luciferase activity in the TLR4-WT + miR-140-5p mimics group was lower than that in the TLR4-WT + mimics NC group $(p<0.05)$. However, the activity of luciferase in the TLR4MUT + miR-140-5p mimics group had not changed compared with the TLR4-MUT + mimics NC group. These results proved that miR-140-5p targeted TLR4.

A

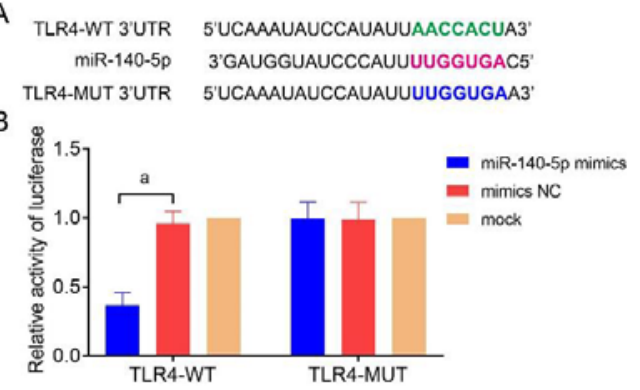

Figure 5: Target relationship between miR-140-5p and TLR4. ${ }^{\text {ap }}<0.05$

\section{DISCUSSION}

MiRNAs are closely with the development of tumors, cardiovascular diseases, diabetes, 
human genetic diseases, and neurological disorders. Currently, the function of miRNAs in morphine tolerance is also receiving increasing attention. Studies show differences in expression of certain miRNAs in morphine-tolerant rats, such as miR-23b, miR-233, miR-190, miR-365 and miR-873a-5p. MiR-873a-5p promotes the development of morphine tolerance in mice by targeting tumor necrosis factor $\alpha$-induced protein 3 gene (A20) [16,17].

Currently, most of the research on miR-140-5p is focused on oncology. MiR-140-5p has been shown to be a tumor suppressor gene, and its expression is reduced in several cancer types [68]. In addition, Liang et al. reported that miR-140$5 p$ is also a tissue-specific miRNA that is predominantly expressed in brain, bone, muscle, and other specific tissues or organs [18]. MiR$140-5 p$ is expressed at low levels in hypoxiaischemia-injured brain tissues, and it was confirmed through animal experiments that miR140-5p exerts cerebral protection through the Wnt/ $\beta$-catenin signaling pathway [19]. Moreover, miR-140-5p levels are increased in brain tissue of Alzheimer's disease patients, and miR-140-5p regulates the expression of sex determining region Y-box transcription factor 2 (SOX2) and Disintegrin and metalloproteinase domaincontaining protein 10 (ADAM10) through inhibiting miR-140-5p, thus representing a method for the treatment of Alzheimer's disease [20]. MiR-140-5p has an essential and complex function in disease, but its specific mechanism varies depending on the type of disease. However, the interrelationship between miR-140$5 p$ and morphine tolerance has not been reported. The results in our study indicate that miR-140-5p expression is down-regulated in spinal cord of the morphine-tolerant rat model, and that miR-140-5p reversed morphine tolerance after injection of miR-140-5p mimics into morphine-tolerant rats. This study revealed that miR-140-5p participates in a novel role in the regulation of morphine tolerance.

TLR4 is an innate immune receptor that is involved in human immune regulation and inflammatory response. Morphine binds to myeloid differentiation factor-2 (MD-2) on TLR4 to activate the TLR4 signaling pathway, which in turn triggers NF-KB activation and production of pro-inflammatory factors, ultimately leading to the development of morphine tolerance [10]. In this study, by bioinformatics website tools, miR-140$5 p$ was predicted to bind complementarily to the 3'-UTR region of TLR4, and Dual-luciferase reporter assay results verified that miR-140-5p has a targeted regulatory effect on TLR4. In addition, injection of $\mathrm{miR}-140-5 \mathrm{p}$ mimics into morphine-tolerant rats decreased TLR4 expression and attenuated their morphine tolerance.

\section{CONCLUSION}

MiR-140-5p inhibits morphine tolerance in rats by targeting TLR4. Haghjooy-Javanmard et al reported that high concentrations of morphine promoted expression of the TLR4 gene, which is similar to our results [21]. This study has provided a preliminary understanding of the downstream mechanisms by which miR-140-5p is involved in the formation of morphine tolerance, and can inhibit morphine tolerance in rats by targeting $T L R 4$.

\section{DECLARATIONS}

\section{Conflict of interest}

No conflict of interest associated with this work.

\section{Availability of data and materials}

All data generated or analyzed during this study are included in this published article.

\section{Contribution of authors}

We declare that this work was done by the authors named in this article and all liabilities pertaining to claims relating to the content of this article will be borne by the authors. Guihua Liu designed the study and supervised the data collection; Ying Xiong analyzed and interpreted the data; Guihua Liu and Ying Xiong prepared the manuscript for publication and reviewed the draft of the manuscript. All authors read and approved the manuscript.

\section{Open Access}

This is an Open Access article that uses a funding model which does not charge readers or their institutions for access and distributed under the terms of the Creative Commons Attribution License (http://creativecommons.org/licenses/by/ 4.0) and the Budapest Open Access Initiative (http://www.budapestopenaccessinitiative.org/rea d), which permit unrestricted use, distribution, and reproduction in any medium, provided the original work is properly credited.

\section{REFERENCES}

1. Fallahi B, Beiki D, Khodaparast MB, Gholamrezanezhad A, Eftekhari M. Additive Clinical Value of Bone Scintigraphy in Patients with Malignant Tumors with

Trop J Pharm Res, September 2021; 20(9): 1885 
Absence of Localized Bone Pain: A Report on Most Common Sex-Related Cancers. Pol Arch Med Wewn 2011; 19(1):51-58.

2. Micheli L, Lucarini E, Corti F, Ciccocioppo R, Calò G, Rizzi A, Ghelardini C, Cesare Mannelli LD. Involvement of the N/OFQ-NOP system in rat morphine antinociceptive tolerance: are astrocytes the crossroad? Eur J Pharmacol 2018; 823: 79-86.

3. Popik P, Kozela E, Danysz W. Clinically available NMDA receptor antagonists memantine and dextromethorphan reverse existing tolerance to the antinociceptive effects of morphine in mice. Naunyn Schmiedebergs Arch Pharmacol 2000; 361(4): 425-432.

4. Wang $Y$, Ji D, Chen G. MiR-379 inhibits proliferation and induces apoptosis in multiple myeloma by targeting $Y$ box binding protein 1. Trop J Pharm Res 2020; 19(9): 1843-1849.

5. He Y, Yang C, Kirkmire CM, Wang ZJ. Regulation of opioid tolerance by let-7 family microRNA targeting the mu opioid receptor. J Neurosci 2010; 30(30): 1025110258.

6. Yan X, Zhu Z, Xu S, Yang LN, Liao XH, Zheng M, Yang D, Wang J, Chen D, Wang L. MicroRNA-140-5p inhibits hepatocellular carcinoma by directly targeting the unique isomerase Pin1 to block multiple cancer-driving pathways. Sci Rep-UK 2017; 7(1): 45915.

7. Fang ZX, Hong SQ, Li TS, Wang J, Jiang L. Genetic and phenotypic characteristics of SCN1A-related epilepsy in Chinese children. Neuroreport 2019; 30(9): 671-680.

8. Flamini V, Dudley E, Jiang WG, Cui Y. Distinct mechanisms by which two forms of miR-140 suppress the malignant properties of lung cancer cells. Oncotarget 2018; 9(92): 36474.

9. Qu J, Tao XY, Teng P, Zhang Y, Guo CL, Hu L, Qian YN, Jiang CY, Liu WT. Blocking ATP-sensitive potassium channel alleviates morphine tolerance by inhibiting HSP70-TLR4-NLRP3-mediated neuroinflammation. J Neuroinflammation 2017; 14(1): 228.

10. Lewis SS, Hutchinson MR, Rezvani N, Loram LC, Watkins LR. Evidence that intrathecal morphine-3glucuronide may cause pain enhancement via toll-like receptor 4/MD-2 and interleukin-1 $\beta$. Neuroscience 2010; 165(2): 569-583.
11. Wang S, Cui Y, Xu J, Gao H. miR-140-5p Attenuates Neuroinflammation and Brain Injury in Rats Following Intracerebral Hemorrhage by Targeting TLR4. Inflammation 2019; 42(5):1869-1877.

12. Elmasry A, Aladeeb NM, Elkaref A, Aboulfotouh N. MiR140-5p inhibits synovial fibroblasts proliferation and inflammatory cytokines secretion through targeting TLR4. Biomed Pharmacother 2017; 96: 208-214.

13. Xu F, Li T, Zhang B. An improved method for protecting and fixing the lumbar catheters placed in the spinal subarachnoid space of rats. J Neurosci Meth 2009; 183(2): 114-118.

14. National Research Council. Nutrient requirements of dairy cattle: 2001[M]. National Academies Press, 2001.

15. Kanda H, Kobayashi K, Yamanaka H, Noguchi K. COX-1dependent prostaglandin D2 in microglia contributes to neuropathic pain via DP2 receptor in spinal neurons. Glia 2013; 61(6): 943-954.

16. Dan D, Yao L, Rui Z, Na S, Yan S, Yang X, Zhang X, Tao $J$, Liu T, Hu L. Protective effects of flavonoids from Coreopsis tinctoria Nutt. on experimental acute pancreatitis via Nrf-2/ARE-mediated antioxidant pathways. J Ethnopharmacol 2018; 224: 261-272.

17. Huang J, Liang X, Wang J, Kong Y, Zhang Z, Ding Z, Song Z, Guo Q, Zou W. miR-873a-5p Targets A20 to Facilitate Morphine Tolerance in Mice. Front NeurosciSwitz 2019; 13:347.

18. A HBL, B JRH, A XQT, A KQD, A GYY, Yu ZC, A LLZ. MicroRNA-140-5p: A novel circulating biomarker for early warning of late-onset post-stroke depression. J Psychiatr Res 2019; 115: 129-141.

19. Han XR, Wen X, Wang YJ, Wang S, Zheng Y-L. MicroRNA-140-5p elevates cerebral protection of dexmedetomidine against hypoxic-ischaemic brain damage via the Wnt/ $\beta$-catenin signalling pathway. J Cell Mol Med 2018; 22(6): 1327.

20. Akhter R, Shao Y, Shaw MK, Formica S, Bekris LM. Regulation of ADAM10 by miR-140-5p and potential relevance for Alzheimer's Disease. Neurobiol Aging 2017; 63: 110-119.

21. Haghjooy-Javanmard S, Ghasemi A, Laher I, Zarrin B, Dana N, Vaseghi G. Influence of morphine on TLR4/ NF-kB signaling pathway of MCF-7 cells. Bratisl Med J 2018; 119(4): 229-233. 